Egyptian Journal of Aquatic Biology \& Fisheries

Zoology Department, Faculty of Science,

Ain Shams University, Cairo, Egypt.

ISSN $1110-6131$

Vol. 25(4): 1085 - 1098 (2021)

www.ejabf.journals.ekb.eg

\title{
Morphological, molecular and clinical assessment of different Anisakis species infecting horse Mackerel Trachurus trachurus from South Mediterranean
}

\author{
Alaa Eldin Eissa ${ }^{1}$, Mohamed Abdelsalam ${ }^{1}$, Marwa M. Attia ${ }^{2}$, Gehad A. Ismail ${ }^{3}$, \\ Rehab A. Qorany ${ }^{4}$, Abdelbary Prince ${ }^{5}$, Rabia A. El Zlitne ${ }^{6}$ and Dalia A. Abdel-moneam ${ }^{1, *}$ \\ ${ }^{1}$ Department of Aquatic Animal Medicine and Management, Faculty of Veterinary Medicine, \\ Cairo University, Egypt. \\ ${ }^{2}$ Department of Parasitology, Faculty of Veterinary Medicine, Cairo University, Egypt. \\ ${ }^{3}$ Agriculture Res. Centre, Animal Health Res. Institute, Fish Diseases Res.. Depart., Egypt. \\ ${ }^{4}$ Agriculture Res. Centre, Animal Health Res. Institute, Fish Diseases Depart., Ismailia, Egypt. \\ ${ }^{5}$ Department of Biochemistry, Faculty of Vet. Medicine, Cairo University, Egypt. \\ ${ }^{6}$ Department of Preventive Medicine, Faculty of Veterinary Medicine, Univ. of Tripoli, Libya \\ "Corresponding Author: dr.daliaashraf@gmail.com
}

\section{ARTICLE INFO}

Article History:

Received: June 11, 2021

Accepted: July 28, 2021

Online: Aug. 30, 2021

Keywords:

Atlantic horse mackerel;

Anisakidae;

A.simplex type1;

A. pegreffii;

Anisakis simplex/

pegreffii hybrid;

Zoonotic parasites.

\section{ABSTRACT}

This study aimed to identify and characterize the most common Anisakis larvae infecting Atlantic horse Mackerel (Trachurus trachurus) in South Mediterranean basin; based on morphological and advanced molecular characterization. Also, the linkage between prevalence of Anisakis spp. larvae and some biological variables including Mackerel age and sex were also investigated. Moreover, the clinical intensity of larvae infection in different organs of infected Mackerel was assessed. Atlantic horse mackerel collected from the southern Mediterranean shores were found to be infected with third stages larvae (L3) of Anisakis species. The Anisakis larvae were found encapsulated as coiled tightly in different fish organs and uncoiled freely in the abdominal cavity of infected $T$. trachurus. These larvae were categorized into 3 types of Anisakis spp. as A. simplex type1; A. pegreffii; Anisakis simplex / pegreffii hybrid based on their morphological and molecular characterizations. Subsequently, the phylogenetic analysis of ITS region of different investigated larvae confirmed the identification of collected Anisakis spp. The remarkably high intensity of Anisakis spp. larvae in Mackerel gonads warns of possible future deleterious impacts on the growth, development and sustainability of Mackerel fisheries at the south Mediterranean coasts.

\section{INTRODUCTION}

The Atlantic horse mackerel Trachurus trachurus (Linnaeus, 1758) is an important fish which is widely distributed throughout the tropical and subtropical aquatic environments worldwide. Mackerel belongs to Perciformes, genus Trachurus and the family Carangidae (Karaoglu and Belduz, 2011). T. trachurus is very delicacy and commercially important fish harvest for fishermen and consumers in North African region. Horse mackerel is a 
migratory and schooling species (Yankova et al. 2009). The juveniles feed on zooplankton while the adults feed on Eggs, larvae, squids and small fish (Yankova $\boldsymbol{e t}$ al. 2009). Such feeding behavior could be the main initiating factor to their infection with helminths during different stages of life cycle. Moreover, their long-life span is another detrimental factor increasing their susceptibility to helminths infections. Anisakids larvae could be recovered from different species of Mackerel. Therefore, eating Mackerel was the primary cause of human Anisakiasis worldwide (Abdelsalam et al. 2020).

Anisakis is one the most important zoonotic nematodes infecting marine fishes worldwide (Abdelsalam et al. 2020). This group of nematodes is commonly named Anisakids and belongs to the super-family: Ascaridioidea, family: Anisakidae and Raphid-ascarididae (Morsy et al. 2015; Arafa et al. 2019). Anisakis simplex is a zoonotic parasitic nematode infecting more than 200 fish species worldwide (Eissa et al. 2015; Eissa et al. 2018; Abdelsalam et al. 2020). Therefore, the infection of fish with anisakid nematodes has posed critical health threats to marine fish consumers, especially when raw or under-cooked infected fish was consumed by humans (Sakanari and McKerrow, 1989). The pathogenesis of $A$. simplex larvae depends upon its ability to penetrate the mucous membrane of the digestive organs such as throat and stomach (Sakanari and McKerrow, 1989). In human they might migrate to different vital organs causing misdiagnosed tumor like masses. The clinical symptoms of the human anisakiasis include nausea, diarrhea; vomiting epigastric pain, and fever, which may occur from 1 to $12 \mathrm{~h}$ after infection. A. simplex also induces both gastric and allergic symptoms (Audicana et al. 2002). Therefore, anaphylactic shock could be expected when human re-infected with anisakis even following cooking (Eissa et al. 2018).

Anisakis spp. possess complex life cycles utilizing number of hosts throughout the course of their life including intermediate hosts such as crustaceans, euphausiid, cephalopods, and fish and final hosts such as cetaceans. The most common stage present in fishes is L3 or third larval stage. The final hosts get the infection by engulfing infected fish and infected intermediate hosts. In the final host, the Anisakis larvae rapidly grow to the larval stage 4 (L4) and then to adults (Pozio, 2013). The adult of Anisakis exist in the gastrointestinal tract of marine mammals and cetaceans (McClelland, 2002).

The Anisakiasis status in south Mediterranean basin remains indistinct due to lack of epidemiological studies in fish and human. Few studies concerning the incidence of Anisakidae in the Mediterranean Sea fishes were performed in Egypt (Morsy et al. 2017; Abou Zaid et al. 2018). In addition, the ichthyoparasitological studies are still poorly investigated in horse mackerel in south Mediterranean basin. The aim of this study is to characterize the most common anisakids species infecting Atlantic horse Mackerel in South Mediterranean basin; based on morphological and advanced molecular characterization. In addition, the impact of age and sex of horse mackerel on the prevalence of Anisakis larvae was also investigated. Finally, the intensity of larvae infection in different organs of infected fish species was studied. 


\section{MATERIALS AND METHODS}

\subsection{Fish Sampling}

Two hundred and forty (240) specimens of Atlantic horse Mackerel were haphazardly collected from local fishermen and retail markets along the southern Mediterranean coasts. The fishes classified into 131 male and 109 females. The collected fishes also; classified according to its age into (3-Month-old: 84; 4 months - old: 95; 5 - months old: 15; 6 months -old: 46).

The collected samples were transported in ice boxes to the laboratories of Aquatic Animal Medicine and Management Lab (AAMML) at the Faculty of Veterinary Medicine, Cairo University. The collected fish were cleaned with saline solution $(0.9 \%)$ for cleansing out the fish from sea weeds, mud and other contaminants. The examined fishes were opened from left side to expose all organs according to Eissa et al. (2018); Attia et al. 2021. Abdominal cavity and internal organs were examined carefully for presence of any anisakids nematodes larvae either free or encapsulated. Intestinal tract was longitudinally opened throughout the entire length and examined with a dissecting microscope for detection of Anisakis spp.. The clinical and postmortem finding of infected fish with anisakids nematodes were recorded and photographed.

\subsection{The impact of age and sex of infected fish on the prevalence of Anisakis spp.}

Intensity was assessed through counting the number of Anisakis larvae in each examined organ.

\subsection{Identification of the collected nematoda}

The collected larvae either encapsulated or free; were rinsed with phosphate buffered saline ( $\mathrm{pH}$ 7.2) and kept for relaxation at $4{ }^{\circ} \mathrm{C}$. after that, they were cleared in lactophenol and mounted with glycerin jelly then examine under stereomicroscope with optical digital USB camera connected computer (Abdelsalam et al. 2020). The anisakids larvae were identified based on the standard morphological criteria described by Eissa $\boldsymbol{e t}$ al. (2018) and Abdelsalam et al. (2020); utilizing the morphological characteristics of the lips surrounding the anterior end, the shape of the esophageal ventriculus; the presence or absence of a boring tooth, the shape of the postanal tail and its mucron or spine. All taken measurements were displayed in millimeters. Another fully identified anisakid nematodes (identification was done under dissecting microscope) were preserved in absolute ethanol (Farjallah et al. 2008; Quiazon et al. 2009; Attia et al. 2021a) for molecular characterization.

\subsection{Molecular characterization of the collected anisakids larvae}

Briefly, the procedures combined the standard PCR for amplification of Internal Transcribed Spacer using the following primers NC2: (5'TTAGTTTCTTTTCCTCCGCT-3') and NC5(5'-GTAGGTGAACCTGCGGAAGGATC ATT-3') as described by Zhu $\boldsymbol{e t}$ al. (1998), and Abdelsalam $\boldsymbol{e t}$ al. (2020). The ITS PCR amplicons were excised from the gel, and the DNA was extracted from the gel using GF- 
1 AmbiClean kit (Vivantis, Malaysia) and sequenced using cycle sequencing PCR reaction with Big- Dye_ Terminator v3.1 Kit (AB-Applied Bioscience), then sequenced in four-capillary ABI PRISM_ 3100-Avant Genetic Analyzer. The chromatogram files were displayed and manually edited using ChromasPro version 2.1.6 software (Technelysium, Australia). Subsequently, BLAST search on NCBI (http://www.ncbi.nlm.nih.gov/pubmed) and Clustal W multiple sequence alignment was applied for the examined consensus sequences to compare with the previously published sequences (Mahmoud et al. 2021). Phylogenetic analysis of obtained sequences was performed using the neighbor-joining method with 1000 bootstrap replications of MEGA 7 according to (Kumar et al. 2016; Attia et al. 2021b). Anisakis berlandi was chosen as the out group.

\section{RESULTS}

\subsection{Clinical findings}

The examined fish were either apparently normal fish or those showing various clinical findings (from mild to severe lesion) such as hemorrhages in different fins, eroded fins, and detached scales (Fig. 1).

\subsection{Postmortem findings}

The infected fish had several lesions in different organs as congestion in stomach, intestine, pyloric caeca and gonads. Visceral adhesions and presence of live nematodes in the organs was the most common postmortem findings. In addition, the anisakids larvae were encapsulated and coiled on the serosal layers of internal organs or lie free in the abdominal cavity of infected fish.

\subsection{Identification of Anisakis species larvae (L3)}

The 3rd stage larvae of Anisakis spp were collected either free or encapsulated in a creamy colored capsule; the larvae were found in most organs of fish as liver; intestine; abdominal cavity. The body is cylindrical and non-segmented but had striation on the cuticle (Table 1). The collected Anisakid spp. were identified as: Anisakis pegreffii; Anisakis simplex and Anisakis simplex / pegreffii hybrid.

The length of ventriculus is the main distinguishing point between $A$. pegreffii and $A$. simplex whereas; in A. pegreffii $0.48-0.79(0.68 \pm 0.27)$ while in A. simplex $0.85-1.7$ $(1.28 \pm 0.54)$. The ventriculus length in the hybrid was very short in comparison with the previous collected anisakids $0.25-0.54(0.26 \pm 0.18)$. All measurements were recorded in Table 1. All Anisakis spp. larvae were cylindrical in shape, their bodies were attenuated at the two ends, the length and width of the whole body of the three recorded species present in Table 1. The anterior end is surrounded by inconspicuous lips, with presence of boring tooth. The larva has esophagus composed mainly of two parts, glandularventriculus part and the muscular part with an oblique junction in esophago-intestinal part. The posterior extremity of the body is provided with short mucron; all measurements of the three species recorded in Table 1. 


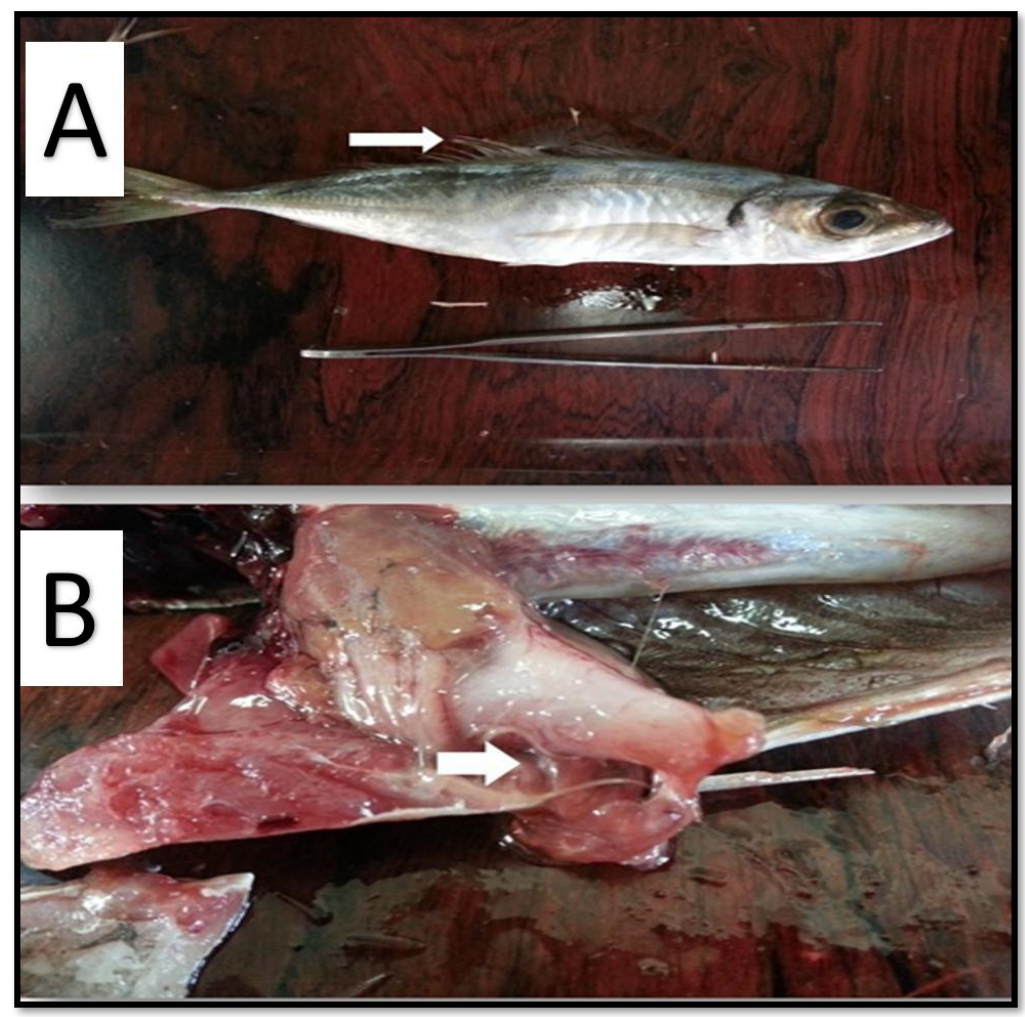

Fig. 1. Clinical finding and postmortem examination of Atlantic horse Mackerel; A: showing erosions in dorsal fin and above the lateral line( referred by arrow) in Atlantic horse Mackerel. B: Mild congestion in stomach, intestine, pyloric and severe congestion in musculature due to Anisakid nematoda which referred by arrow. (Anisakid nematoda referred by arrow).

Table 1. Morphological criteria of third stage larvae (L3) of Anisakis species reported in Atlantic horse mackerel caught from Mediterranean shores.

\section{Morphological characters}

Anisakis simplex

$17.9-23.6(18.6 \pm 1.5)$
$0.55-0.9(0.75 \pm 0.45)$
$0.4-0.55(0.47 \pm 0.07)$
$1.7-3.9(2.95 \pm 0.55)$
$0.85-1.7(1.28 \pm 0.54)$.
$0.25-0.38(0.29 \pm 0.10)$
$0.15-0.19(0.16 \pm 0.07)$
$0.029-0.040(0.034 \pm 0.04)$

Anisakis pegreffii

$11.10-26.78(17.24 \pm 11.08)$

$0.38-0.60(0.47 \pm 0.155)$

$0.20-0.31(0.25 \pm 0.07)$

$1.04-2.11(1.57 \pm 0.75)$

$0.48-0.79(0.68 \pm 0.27)$

$0.12-0.27(0.18 \pm 0.10)$

$0.05-0.12(0.07 \pm 0.04)$

$0.02-0.03(0.024 \pm 0.007)$
Anisakis simplex/pegreffii

hybrid

$9.00-17.5(12.5 \pm 6.01)$
$0.25-0.56(0.37 \pm 0.21)$
$0.19-0.23(0.21 \pm 0.02)$
$0.56-1.75(0.98 \pm 0.84)$
$0.25-0.54(0.26 \pm 0.18)$.
$0.12-0.20(0.15 \pm 0.05)$
$0.04-0.09(0.06 \pm 0.03)$
$0.01-0.03(0.02 \pm 0.014)$

Data expressed as min- max (average \pm SE) $\mathrm{mm}$. 


\subsection{Sequence analysis of ITS region}

An approximately $1000 \mathrm{bp}$ fragment of the ITS region of Anisakis larvae type I was yielded by using the universal primer NC5 and NC2. Four purified PCR products of ITS regions were directly sequenced to identify the species. The accession numbers of ITS regions of Anisakis spp. larvae were MW507186, MW507187, MW507188 and MW507189. The accession numbers (MW507186 and MW507187) revealed 99.88\% identity with $A$. pegreffii (LC536533-LC536532-Japan), with one nucleotide insertion in the Egyptian samples. On the other hand, the accession number (MW507188) exhibited 99.88\% identity with A. simplex (MT516319-Spain) and (LC536534-Japan), with one nucleotide differences. While (MW507189) showed $99.88 \%$ identity with Anisakis simplex/ Anisakis pegreffii hybrid (KF032056-Turkey) and (JN005768-Portugal), with one nucleotide differences.

Remarkably, the neighbor-joining phylogenetic tree of amplified sequences of $\mathrm{A}$. pegreffii, A. simplex, and A. simplex x A. pegreffii hybrid were grouped with known sequences of the relevant sequences of anisakidae species and separated from each other. The clade of present A. pegreffii sequences grouped with A. pegreffii from Japan, Thailand, China and Spain. On the other hand, A. simplex sequence in this study grouped with A. simplex from Japan, Spain, Turkey, Portugal, Germany and Turkey. Furthermore, A. simplex x A. pegerffii hybrid sequence grouped with A. simplex x A. pegerffii hybrid from Turkey, Germany, and Portugal. (Fig. 2).

\subsection{Impact of Atlantic horse mackerel sex on the prevalence of Anisakis spp.}

The percentages of infected females (26\%) were relatively higher than males $(21 \%)$. The total number of Anisakis larvae in female fish was 973 while the number was 615 in males (Table 2). Statistical analysis indicated that there were significant differences in total count of 3rd stage larvae among examined fishes between male and female seasons at probability ratio $\mathrm{Pr}=0.04$.

\subsection{Impact of age factor on the prevalence of Anisakis spp. infection}

The 240 fish samples were categorized according to age into 3, 4, 5 and 6 years. The statistical analysis indicated that there was a significant difference $(\operatorname{Pr}=<0.0001)$ age / infection level. This obviously indicated that the older fish got the higher number of larvae in their bodies. In the 3 years old examined fish, a total of $5 \%$ of fish sample has third stage larvae. In the 6 years old fish, the prevalence of third larval stage was $56 \%$ which is remarkably higher than the 3 years age category. (Table 3 ).

\subsection{The intensity of Anisakis spp. larvae (L3) in different fish organs}

A total number of 1588 of larvae were collected from 55 infected fish out of 240 examined Atlantic horse mackerel samples. A total of 299, 264, 221, 100, 19 and 685 were collected from stomach, intestine, pyloric caeca, muscles, abdominal cavity and gonads respectively. The total number of Anisakis larvae was much higher in ovaries 438 than testis 247. All five organs (gonads, stomach, intestine, pyloric caeca and abdominal cavity) were infected with L3 stage of Anisakis species at different intensities and there 
was significantly difference $(\mathrm{Pr}=<0.0001)$. The highest intensity of infection was in ovaries $(28 \%)$.

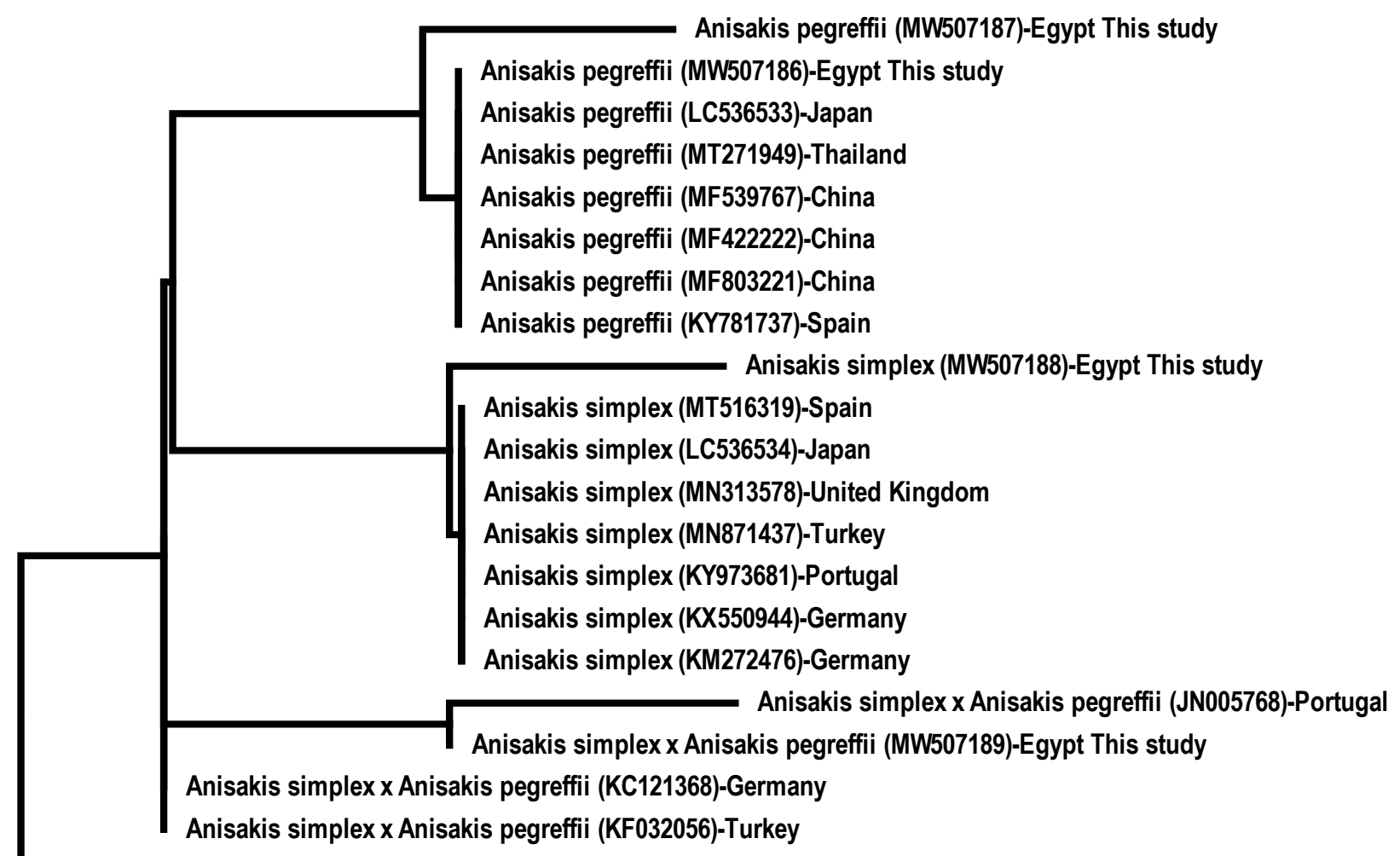

Anisakis berlandi (MH481357)-Mexico

\section{5}

Fig. 2. Neighbor-Joining Phylogenetic tree showed the comparative analysis of ITS region sequences of Anisakis simplex, A. pegreffii and A. simplex x A. pegreffii hybrid identified in this study with other closely related Anisakis spp.

Table 2. Prevalence of the retrieved third stage larvae (L3) of different Anisakis species among examined male and female Atlantic horse mackerel.

\begin{tabular}{llllc}
\hline Anisakis spp & Sex & No. infected fish & No. of retrieved larvae & Prevalence\%* \\
\hline M & 27 & 615 & 21 \\
& F & 28 & 973 & 26 \\
\hline
\end{tabular}

*Represent percentage of infection in relation to the total number of collected fish.

M: Male: number of collected male fish $=131$

F: Female: number of collected male fish $=109$ 
Table 3. Prevalence of retrieved third stage larvae of Anisakis species infected in examined Atlantic horse mackerel through different ages.

\begin{tabular}{lcccc}
\hline Anisakis spp. & $\begin{array}{c}\text { Age of collected } \\
\text { fish }\end{array}$ & $\begin{array}{c}\text { No. of infected } \\
\text { fish }\end{array}$ & No. of larvae & Prevalence\%* \\
\hline Anisakis & 3 & 4 & 22 & 5 \\
species & 4 & 17 & 407 & 18 \\
& 5 & 8 & 194 & 53 \\
& 6 & 26 & 965 & 56 \\
\hline
\end{tabular}

*Represent percentage of infection in relation to the total number of collected fish.

No. of collected fishes at different age (3 Month: 84; 4 months: 95; 5 months: 15; 6 months: 46).

\section{DISCUSSION}

The Atlantic horse mackerel fish is an economically and commercially important fish harvest. Thus, studying the health, growth and fecundity of this valuable fish species is of great interest for aquatic veterinarians at Mediterranean basin. The occurrence of zoonotic nematodes is increasing among Mediterranean countries as a result of dumping of sewage directly into the Mediterranean Sea (Fernandez-Jover et al. 2010). Several Anisakis spp. has been documented infecting marine fishes among southern countries of Mediterranean basin including Morocco, Tunisia and Finally Libya (Farjallah et al. 2008; Eissa et al. 2015). Anisakis spp including A. simplex, A. typica, and A. pegreffii, infected several commercial fish inhabiting the Mediterranean Sea. Literatures from the Southern Mediterranean countries have proved that horse mackerel is commonly found to be infected with Anisakids larvae (Manfredi et al. 2000; Abdelsalam et al. 2020).

In the current study, the identification of anisakid recovered from horse mackerel were coincided with the criteria of $A$. pegreffii and $A$. simplex complex as described by (Eissa et al. 2018; Abdelsalam et al. 2020). A total of 904 A. pegreffii larvae, 440 Anisakis simplex larvae, and 244 Anisakis simplex/ Anisakis pegreffii hybrid larvae were identified from a total of 1588 Anisakid nematodes which were collected from 240 Atlantic horse Mackerel throughout spring and summer seasons. For those identified as A. pegreffii, the recorded taxonomical measures were the total length / width of the ventriculus, esophageal length, and body length were shorter than those documented for A. simplex and slightly larger than A. typica as described in table (2) (Quiazon et al. 2009; Eissa et al. 2015). On the contrary, those clusters assigned as "Anisakis simplex / pegreffii hybrid genotype. The main difference point between Anisakis simplex and A. pegreffii is the length of ventriculus, body length (Davey, 1971; Mattiucc et al. 2005). There is a clear difference was observed in the ventriculus length between A. simplex and A. pegreffii. The main criteria that discriminated between Type I and Type II Anisakis larvae was the ventriculus length whereas in type I ventriculus length $(0.65-1.5 \mathrm{~mm})$ while in type II 
$(0.52-0.75 \mathrm{~mm})$; while the A. pegreffii the ventriculus length $0.50-0.78 \mathrm{~mm}$ but have mucron that the Anisakis type II did not have this mucron (Quiazon et al. 2008). This study proved the importance of the combination of genetic and morphometric tools for accurate identification and differentiation between different Anisakis spp. The sequencing of ITS regions demonstrated its usefulness in detecting and distinguishing $\mathrm{A}$. pegreffi from A. simplex and A. simplex x A. pegreffii hybrid. In this study, the A. pegreffii of this study grouped with other A. Pegreffii samples and separated from A. simplex and A. simplex x A. pegreffii hybrid. The present A. pegreffii, A. simplex and A. simplex $x$ A. pegreffii hybrid displayed the highest similarity percentages with other relevant A. pegreffii, A. simplex and A. simplex x A. pegreffii hybrid, respectively. Accordingly, morphometeric and genetic evidence proved that these nematodes larvae belonging to the family Anisakidae and could be identified as A. pegreffii, A. simplex, and A. simplex x A. pegreffii infecting the Egyptian marine fishes.

Pathologically, Anisakis larvae utilize the triacylglycerols (TAG) deposited in fish muscle during their course of invasion/ migration. The depletion of TAG affect swimming capability of the infected fishes due to loss of energy with consequent general weakening and high liability to predation. Pathologically, the loss of the lipid deposit from the fish muscles facilitates the adhesions of visceral organs that result in granulomatous inflammatory responses with the modification of fish body shape with an ultimate poor health condition and poor growth (Mika et al. 2010; Santoroa et al. 2010). The feeding behavior of horse mackerel allows them to contract larval Anisakis species from the infected mollusks and marine mussels with the early larval stages of anisakids. The early stage of Anisakis larvae developed into 3rd larval stage which can penetrate the intestinal wall to abdominal cavity and then to visceral organs with high tendency to gonads, kidney and spleen. The migration of the 3rd larval stages corrupted the gastric mucosal epithelia leading to poor digestion and absorption with ultimate poor growth rates (Eissa et $\boldsymbol{a l}$. 2015). The sex as a variable has proved an influential effect on the prevalence of Anisakis spp. infection among different examined Atlantic horse Mackerel. The percentage of infection was slightly higher in females $(26 \%)$ than males $(21 \%)$. These results relatively agreed with similar study published by Qasim and Ayub, (2012). These results coincided with that of Carvajal et al. (1979). However, another study published by Ozer, (2000) has revealed that there are no differences in infection rate between male and female fish.

Moreover, George-Nasciment et al. (1983) have recorded that sexually mature females had significantly higher prevalence of infections with Anisakis spp. than had males in the Chilean Jack Mackerel ( $\mathrm{P}<0.05)$. The relative higher prevalence of nematode infections in females than males could be attributed to relative hormonal stress during the maturity and spawning periods in females. Generally, mature females are liable to diseases than males due to immunosuppressive nature of stress hormones (Corticosterols) that is known to be much higher in mature spawning females than males rendering them to be 
vulnerable to infections than others (Eissa et al. 2015). Another interesting factor is the stomach fullness extent in females is much higher than males. This could allow females to apprehend larger numbers of larvae in their food than males with consequent increase in infection prevalence as well as intensity within fish organs. This hypothesis is supported by Juras and Yamaguti, (1985) study who declared that stomach fullness of females was higher than that of the males and immature fishes.

The prevalence of Anisakis spp. infection among Atlantic horse Mackerel is also variable according to the length / age of the examined fish. Previous studies have indicated that the prevalence of 3rd stage larvae of Anisakis simplex was highest in fishes with length 30-34 cm (6 years old) compared to other length / age categories (Abaunza et al. 1995; Adroher et al. 1996). In our study the 240 examined fish samples were categorized according to age into 3, 4, 5- and 6-years categories. The statistical analysis presented a significant difference $(\operatorname{Pr}=<0.0001)$ considering the age / infection level variable. The results indicated that the older the fish the higher the number of larvae in their bodies and the maximum infection percentage was in the 6 years category (56\%). This higher infection prevalence could be attributed to the prolonged durations of exposure and predation along the long lifetime of such category. This hypothesis consistently agreed with that declared by Smith, (1984) who stated that the number of ascaridoid larvae that accumulate in marine fish is proportional with age, predatory behavior and increased feeding rates. Similar observations and explanations have been presented by Smith and Wootten, (1978); Manfredi et al. (2000).

The results also revealed that all examined five organs (gonads, stomach, intestine, pyloric ceca and abdominal cavity) were infected with L3 stage of Anisakis spp. at different intensity levels with noticeable significant difference $(\operatorname{Pr}=<0.0001)$. The highest intensity of infection was recorded in examined ovaries $(28 \%)$. This result was consistent with that of Tantanasi et al. (2012) who have reported that most of L3 stage of Anisakis spp. retrieved from Atlantic horse mackerel were found in the gonads rather than on other organs.

\section{CONCLUSION}

In conclusion, Anisakid infection would present a vulnerable biological obstacle to the development and sustainability of Mackerel fisheries by their direct impact on growth, fertility and fecundity of both male and female fish. Moreover, their drastic impacts on intestinal, abdominal and gonadal health might be another critical factor that poses an indirect threat to the vital physiological process with consequent disruption of the fish's internal homeostasis. The sum of these biological growth, fecundity and health disruptors is an abundant decline of the mackerel fisheries through the southern coasts of the Mediterranean Sea. Ultimately, these highly invasive anisakid larvae represent a vulnerable threat to human health by their high zoonotic capability. 


\section{REFERENCES}

Abaunza, P.; Vellamor, B. and Perez, J.R. (1995). Infestation by larvae of Anisakis simplex (Nematoda: Ascaridata) in horse mackerel, Trachurus trachurus, and Atlantic mackerel, scomberscombrus, in ICES divisions VIIIb, VIIIc and IXa (NNW of Spain). SCI. MAR, 59 (3-4): 223-233.

Abdelsalam, M.; Attia, M. M. and Mahmoud, M. A. (2020). Comparative morphomolecular identification and pathological changes associated with Anisakis simplex larvae (Nematoda: Anisakidae) infecting native and imported chub mackerel (Scomber japonicus) in Egypt. Regional Studies in Marine Science 39 (2020) 101469.

Abou Zaid, A.; Bazh, E.; Desouki, A. et al, (2018). Metazoan parasite fauna of wild sea bass: Dicentachus labrax (Linaeus,1758) in Egypt. Life Sci. J. 15, 6:48-60.

Adroher, F.J.; Valero, A.; Ruiz-Valero, J. and Iglesias, L. (1996). Larval anisakids (Nematoda: Ascaridoidea) in horse mackerel (Trachurus trachurus) from the fish market in Granada (Spain). Parasitol Res (1996) 82:253-256.

Al-Zubaidy, A. B. (2010). Third-stage larvae of Anisakis simplex (Rudolphi, 1809) in the Red Sea fishes, Yemen coast. Marine Scienes, 21(1).

Arafa, W.M. and Hassan, A.H.A., et al. (2019). Occurrence and molecular characterization of zoonotic Anisakis simplex sensu stricto and Anisakis pegreffii larvae in retail-marketed fish. J. Food Saf. 39, 1-7.

Attia, M. M.; Abdelsalam, M.; Korany, R. M. S. and Mahdy, O. A. (2021). Characterization of digenetic trematodes infecting African catfish (Clarias gariepinus) based on integrated morphological, molecular, histopathological, and immunological examination. Parasitology Research https://doi.org/10.1007/ s00436-021-07257-x.

Attia, M.M.; Elgendy, M.Y. and Abdelsalam, M. et al. (2021a). Morpho-molecular identification of Heterophyes heterophyes encysted metacercariae and its immunological and histopathological effects on farmed Mugil cephalus in Egypt. Aquacult Int. https://doi.org/10. 1007/s10499-021-00708-3.

Attia, M.M.; Elgendy, M.Y.; Prince, A.; El-Adawy, M.M. and Abdelsalam, M. (2021b). Morphomolecular identification of two trichodinid coinfections (Ciliophora: Trichodinidae) and their immunological impacts on farmed Nile Tilapia. Aquac Res. https://doi.org/10. 1111/are.15281.

Audicana, M.T.; Ansotegui, I.J.; de Corres, L.F. and Kennedy, M.W. (2002). Anisakis simplex: dangerous dead and alive? Trends Parasitol., 18, pp. 20-25

Carvajal, J.; Cattan, P.E.; Castillo, C.; Schatte, P. (1979). Larval anisakids and other helminthes in the hake, Merluccius gayi (Guichenot) from Chile. J Fish Biol 15: 671-677

Cocheton, J.J.; Cabout, I. and Lecomte, I. (1991). Anisakiase et infections par les 
anisakidés. Ann. Med. Interne; 142: 121-131

Davey, J.T. (1971). The incidence of Anisakis larvae (Nematoda: Ascaridata) in the commercially exploited stocks of herring (Clupea harengus L., 1758) (Pisces: Clupeidae) in British and adjacent waters. J. Fish Biol. 4, 535-554.

EFSA, (2010). EFSA panel on biological hazards (BIOHAZ); scientific opinion on risk assessment of parasites in fishery products. EFSA J. 8 (4), 1543.http://dx.doi.org/10.2903/j.efsa.2010.1543(http://www.efsa.europa.eu, 91 pp.).

Eissa, A.E.; Abdelsalam, M.; Abumhara, A.; Kammon, A.; Gammoudi, F.T.; Ben Naser, K.M.; Borhan, T. and Asheg, A. (2015). First Record of Vibrio vulnificus/ Anisakis pegreffii Concurrent infection in Black scorpionfish (Scorpaenaporcus) from the South Mediterranean Basin. Research Journal of Phamaceutical Biological and Chemical Sciences.6(3). 1537.

Eissa, A.E.; Showehdi, M.L.; Ismail, M.M.; El-Naas, A.S.; Abu Mhara, A.A. and Abolghait, S.K. (2018). Identification and prevalence of Anisakis pegreffii and A. pegreffii X A. Simplex (s.s.) hybrid genotype larvae in Atlantic horse Mackerel (Trachurus trachurus) from some North African Mediterranean coasts. Egypt. J. Aquat. Res. 44, 21-27.

Farjallah, S.; Busi, M.; Mahjoub, M.O.; Slimane, B.B.; Paggi, L.; Said, K. and D'Amelio, S. (2008). Molecular characterization of larval anisakid nematodes from marine fishes off the Moroccan and Mauritanian coasts. Parasitol. Int. 57, 430-436.

Fernandez-Jover, D.; Faliex, E; Sanchez-Jerez, P.; Sasal, P. and Bayle-Sempere, J. (2010). Coastal fish farming does not affect the total parasite communities of wild fish in SW Mediterranean. Aquaculture 300: 10-16.

George-Nasciment, M.A.; Carvajal, J.G. and Alcain, H.C. (1983). Occurrence of Anisakis sp. larvae in the chilean Jack Mackerel, Trachurus murphyi Nichols 1920. RevistaChilena de Historia Natural 56: 31-37.

Guz, L.; Studzińska, M.B.; Sadzikowski, A.B. and Gundłach, J.L., (2005). Anisakiosis. Annales, Universitatis Mariae Curie-Skłodowska. Vol. LX, 10 Sectio.

ICES, (1999). Report of the Horse Mackerel Otolith Workshop, Lowestoft, 15-19 January 1999. ICES, C.M.

Juras, A.A. and Yamaguti, N. (1985). Food and feeding habits of king weakfish Macrodonacylodon(Bloch and Schneider, 1801) caught in the southern coast of brazil (lat, $29^{\circ}$ to $32^{\circ}$ s). Bolm Inst. Oceanogr. 33(2):149-157.

Karaoglu, H. and Belduz, A.O. (2011). Multivariate Discrimination among three Trachurus species from Turkey. Trabzon, Turkey, 10 (1): 121 - 127. 
Kumar, S.; Stecher, G. and Tamura, K. (2016). MEGA7: Molecular evolutionary genetics analysis version 7.0 for bigger datasets. Molecular Biology and Evolution, 33(7), 1870-1874. https://doi.org/10.1093/molbev/msw054.

Manfredi, M.T.; Crosa, G.; Galli, P. and Ganduglia, S. (2000). Distribution of Anisakis simplex in fish caught in the Ligurian Sea. Parasitol. Res. 86, 551-553. DOI: $10.1007 / \mathrm{s} 004360000202$.

Mattiucci, M.; Dailey, S. C.; Webb, N. B.; Barros, R.; Cianchi. and Bullini, L. (2005). Evidence of a new species of Anisakis Dujardin, 1845: Morphological description and genetic relationships between congeners (Nematodes: Anisakidae). Syst Parasitology 61: 157-171.

Mahmoud, A. M.; Attia, M. M.; Abdelsalam, M; Abdel- Moneam, D. A. and Ewiss M. A. Z. (2021). Ergasilus extensus and bacterial co- infection in flathead grey mullet, Mugil cephalus (Linnaeus, 1758), are associated with pathological changes and immunological gene expression alterations. Aquaculture Research. 2021;00:1-9. DOI: 10.1111/are.15476.

McClelland, G. (2002). The trouble with sealworms (Pseudoterranova decipiens species complex, Nematoda): a review Parasitology, 124 (Suppl.), pp. 183-203

Morsy, K.; Bashtar, A.; Mostafa, N.; El Deeb, S. and Thabet, S. (2015). New host records of three juvenile nematodes in Egypt: Anisakis sp. (type II), hysterothylacium patagonense (Anisakidae), and Echinocephalus overstreeti (Gnathostomatidae) from the greater lizard fish Saurida undosquamis of the Red Sea. Parasitol. Res. 114, 1119-1128.

Mika, A.; Gołebiowski, M.; Szafranek, J.; Rokicki, J. and Stepnowski, P. (2010). Identification of lipids in the cuticle of the parasitic nematode Anisakis simplex and the somatic tissues of the Atlantic cod Gadusmorhua. Exp. Parasitol. 124:334-340.

Özer, A. (2000). The occurrence of three species of Trichodina (Ciliophora: Peritrichia) on Cyprinus carpio in relation to culture conditions, seasonality and host characteristics. Acta Protozool., 39, 61-66.

Qasim, S. and Ayub, Z. (2012). Prevalence and intensity of parasites in edible fishes landing at Karachi Fish Harbour. Pakistan Journal of Zoology, 44(6).

Pozio, E. (2013). Integrating animal health surveillance and food safety: the example of Anisakis. Rev Sci Tech, 32(2), 487-96.

Quiazon, K. M. A.; Yoshinaga, T.; Ogawa, K. and Yukami, R. (2009). Morphological differences between larvae and in vitro-cultured adults of Anisakis simplex (sensu stricto) and Anisakis pegreffii (Nematoda: Anisakidae). Parasitology International 57: 483-489.

Quiazon, K.M.A.; Yoshinaga, T.; Ogawa, K. and Yukami, R. (2008). Morphological differences between larvae and in vitro-cultured adults of Anisakis simplex (sensu 
stricto) and Anisakis pegreffii (Nematoda: Anisakidae). Parasitol Int. 2008, 57: 483-489. 10.1016/j.parint.2008.06.003.

Sakanari, J. A., and Mckerrow, J. H. (1989). Anisakiasis. Clinical microbiology reviews, 2(3), 278-284.

Santoroa, M.; Mattiuccia, S.; Paoletti, M.; Liotta, A.; Uberti, B.D.; Galiero, G. and Nascetti, G. (2010). Molecular identification and pathology of Anisakis pegreffii (Nematoda: Anisakidae) infection in the Mediterranean loggerhead sea turtle (Carettacaretta). Vet. Parasitol.174:65- 71.

Serracca, L.; Cencetti, E.; Battistini, R.; Rossini, I.; Prearo, M.; Pavoletti, E.; Fioravanti, M.L.; Righetti, M.; Di Donfrancesco, B. and Ercolini, C. (2013). Survey on the presence of Anisakis and Hysterothylacium larvae in fishes and squids caught in Ligurian Sea. Vet. Parasitol. 196, 547-551.

Setyobudi1, E.K. and Ho Kim, J. (2010). Anisakis simplex (Nematode: Anisakidae) L3 Larvae Infection in Chumsalmon (Oncorhynchus keta) from Namdae River, South Korea. North Pacific Anadromous Fish Commission (NPAFC), 1257. 210 -702.

Shih, H.H. and Jeng, M.S. (2002). Hysterothylacium aduncum (Nematoda: Anisakidae) Infecting a Herbivorous Fish, Siganus fuscescens, off the Taiwanese Coast of the Northwest Pacific. Zoological Studies 41(2): 208-215.

Smith, J.W and Wootten, R. (1978). Anisakis and anisakiasis. Adv. Parasitol., 16: 93163.

Smith. J. W. (1984). The abundance of Anisakis simplex L3 in the body-cavity and flesh of marine teleosts. International journal for parasitology, 14(5): 491-495.

Sonko, P.; Chih-Cheng Chen, S.; Chou,C.; Huang, Y.; , Hsu, S.; Barca'k,D.; Oros, M. and Fan, C. (2019). Multidisciplinary approach in study of the zoonotic Anisakis larval infection in the blue mackerel (Scomber australasicus) and the largehead hairtail (Trichiurus lepturus) in Northern Taiwan. Journal of Microbiology, Immunology and Infection. https://doi.org/10.1016/j.jmii.2019.04.012.

Tantanasi, J.; Diakou, A.; Tamvakis, A. and Batjakas, E. (2012). Anisakis spp. burden in Trachurus trachurus. HELMINTHOLOGIA, 49, 1: 16 - 20.

Valentini, A.; Mattiucci, S.; Bondanelli, P.; Webb, S. C.; Micnucci-Giannone, A. A.; Colom-Llavina, M. M. and Nascetti, G. (2006). Genetic relationships among Anisakis species (Nematoda: Anisakidae) inferred from mitochondrial cox2 sequences, and comparison with allozyme data. Journal of Parasitology 92: 156-166.

Yankova, M.; Pavlov, D. and Raykov, V. (2009). Population Dynamics Of HorseMackerel (Trachurus Mediterraneus), As A Valuable Economic Species For The Bulgarian Black Sea Coast. Annals of the University Dunarea de Jos of Galati Fascicle VI--Food Technology, 3(32).

Zhu, X.; Gasser, R.B.; Podolska, M. and Chilton, N.B. (1998). Characterization of anisakid nematodes with zoonotic potential by nuclear ribosomal DNA sequences. Int. J. Parasitol. 28: 1911-1921. 